\title{
DIFFERENTIAL FEEDBACK OF MIMO CHANNEL CORRELATION MATRICES BASED ON GEODESIC CURVES
}

\author{
Daniel Sacristán ${ }^{1}$, Antonio Pascual-Iserte $e^{1,2}$ \\ ${ }^{1}$ Centre Tecnològic de Telecomunicacions de Catalunya (CTTC) - Spain \\ ${ }^{2}$ Dept. of Signal Theory and Communications - Universitat Politècnica de Catalunya (UPC) - Spain \\ email: daniel.sacristan@cttc.es, antonio.pascual@upc.edu
}

\begin{abstract}
This paper proposes a differential quantization strategy to be used in the feedback link of a multi-input-multi-output (MIMO) communication system. This algorithm is applied to the channel correlation matrix exploiting geodesic curves and the intrinsic geometry of positive definite Hermitian matrices. Simulation results in the paper show that the proposed algorithm improves other techniques based on the direct quantization of the channel response matrix or the quantization of the subspace spanned by the strongest eigenmodes of the MIMO channel, i.e., Grassmannian based techniques. The main drawback of Grassmananian based algorithms is that the transmitter is constrained to apply a uniform power allocation among eigenmodes, which is not forced in the algorithm proposed in this paper.
\end{abstract}

Index Terms-MIMO systems, feedback communication, quantization, differential geometry.

\section{INTRODUCTION}

Multi-input-multi-output (MIMO) communication channels are known to provide significant gains in system performance. These gains depend strongly on the quality of the channel state information (CSI) which is available during the design. Obviously, the best performance is achieved when such CSI is complete and perfect, although this is a not realistic assumption, specially at the transmitter.

In scenarios where channel reciprocity does not hold, a feedback channel with limited capacity can be used to send the CSI from the receiver to the transmitter. In this sense, proper quantization procedures to be applied to the channel estimates have to be designed. Following this idea, [1] (and other works by the same authors) proposed Grassmannian packaging, which is the optimum non-differential quantization strategy for zero-mean Rayleigh MIMO channels with independent components and where the transmitter is constrained to apply a uniform power allocation among the transmission modes. It was shown that, under this constraint, the transmitter only needs to know which is the subspace spanned by the strongest right singular eigenvectors of the MIMO channel matrix $\mathbf{H}$. Based on this, the quantization is applied over the Grassmannian manifold, i.e., the set of all the possible subspaces [2].

In [3] the same constraint was considered but a differential quantization was applied, which makes sense in scenarios where the channel is slowly varying and, therefore, the temporal correlation

This work was partially supported by the Catalan Government under grant 2005SGR-00996; by the Spanish Government under projects TEC2008-06327-C03-01 (FBMC-MULAN) and 2A103 (MIMOWA) from MEDEA+ program (AVANZA I+D TSI-020400-2008-150), and by the European Commission under project NEWCOM++ (216715). can be exploited to improve the quality of the quantization. The proposed technique consisted in defining geodesic curves over the Grassmannian manifold [2], i.e., curves whose all their points are within such manifold, and which connect pairs of points in this space (i.e., subspaces) with a minimum distance.

In more general cases where the power allocation is not constrained to be uniform, the optimum linear signalling scheme depends on the MIMO channel correlation matrix, i.e., $\mathbf{R}_{H}=\mathbf{H}^{H} \mathbf{H}$ for any quality criterion such as mutual information, mean square error (MSE), signal-to-noise ratio (SNR), bit error rate (BER), among others [4]. Note that in this case, the design depends on the right singular vectors of the channel matrix (and not only on the subspace spanned by them) in addition to the eigenvalues. Taking this into account, now the quantization should be applied over the set of correlation-like matrices, i.e., Hermitian and positive definite matrices, instead of the Grassmannian manifold.

In this paper we propose a differential quantization algorithm to be applied to the channel correlation matrix exploiting the intrinsic differential geometry of the set of positive definite Hermitian matrices and using geodesic curves. More concretely, we propose an algorithm that at each iteration defines a set of orthogonal curves in the set and identifies a set of candidate points which are the quantization proposals. The selection of the point to be fed back depends on the cost function, that is related to the specific measure of performance for the system or can be the geodesic distance to the exact channel realization. [5] also used the concept of geodesic curves in the set of correlation-like matrices, but the application was for channel classification instead of differential quantization.

The paper is organized as follows. The system and signal models are given in section 2. Section 3 introduces the geometry of the space of correlation matrices, and then the description of the algorithm follows in section 4. Numerical simulations are shown in section 5, and section 6 concludes the paper.

\section{SYSTEM AND SIGNAL MODELS}

Let us consider a MIMO channel with $n_{T}$ and $n_{R}$ transmit and receive antennas, respectively, represented at time instant $n$ by $\mathbf{H}(n) \in \mathbb{C}^{n_{R} \times n_{T}}$. The $n_{R}$ received signals at the same time instant, assuming a linear transmitter, can be expressed as

$$
\mathbf{y}(n)=\mathbf{H}(n) \mathbf{B}\left(\widehat{\mathbf{R}}_{H}(n)\right) \mathbf{x}(n)+\mathbf{w}(n) \in \mathbb{C}^{n_{R}},
$$

where $\mathbf{x}(n) \in \mathbb{C}^{n_{S}}$ represents the $n_{S}$ streams of signals to be transmitted with $\mathbb{E}\left[\mathbf{x}(n) \mathbf{x}^{H}(n)\right]=\mathbf{I}$, and $\mathbf{B} \in \mathbb{C}^{n_{T} \times n_{S}}$ is the linear transmitter matrix that must satisfy the mean transmit power constraint $\|\mathbf{B}\|_{F}^{2} \leq P_{T}\left(\|\cdot\|_{F}\right.$ stands for the Frobenius norm). Note that we explicitly indicate that the transmitter depends on the available estimate of the channel correlation matrix $\widehat{\mathbf{R}}_{H}(n)$, where the 
exact matrix is $\mathbf{R}_{H}(n)=\mathbf{H}^{H}(n) \mathbf{H}(n)$. The AWGN at the receiver is represented by $\mathbf{w}(n) \in \mathbb{C}^{n_{R}}$ with $\mathbb{E}\left[\mathbf{w}(n) \mathbf{w}^{H}(n)\right]=\sigma_{w}^{2} \mathbf{I}$.

In this paper, we consider that the receiver estimates perfectly the current channel matrix $\mathbf{H}(n)$, as in $[1,3]$, and that the transmitter designs $\mathbf{B}$ assuming that the available CSI at its side represented by $\widehat{\mathbf{R}}_{H}(n)$ is also perfect. In reality, however, the CSI at the transmitter is not perfect because it is a quantized version of the perfect CSI estimated at the receiver. The possibility of errors and delay in the feedback channel is not considered in this paper. The transmitter design can be done according to different criteria, such as the maximization of the mutual information or the SNR, or the minimization of the MSE or the BER, among others. In all the cases, the optimum transmitter has been shown to depend only on the channel correlation matrix $\mathbf{R}_{H}(n)$ [4]. For each of the design criteria a cost function $d\left(\widehat{\mathbf{R}}_{H}(n), \mathbf{H}(n)\right)$ can be defined, so that the design objective is its minimization. A couple of examples of cost functions are given below $^{1}$ (we drop the dependency with respect to the time index $n$ for the sake of clarity in the notation):

- Maximization of the SNR with single beamforming $\left(n_{S}=1\right)$ :

$$
d\left(\widehat{\mathbf{R}}_{H}(n), \mathbf{H}(n)\right)=-\frac{1}{\sigma_{w}^{2}}\|\mathbf{H B}\|_{F}^{2},
$$

where the transmission matrix $\mathbf{B} \in \mathbb{C}^{n_{T} \times 1}$ is defined as

$$
\mathbf{B}\left(\widehat{\mathbf{R}}_{H}(n)\right)=\sqrt{P_{T}} \mathbf{u}_{\max }\left(\widehat{\mathbf{R}}_{H}(n)\right),
$$

and $\mathbf{u}_{\max }(\cdot)$ stands for the unit-norm eigenvector of maximum associated eigenvalue.

- Maximization of the mutual information:

$$
d\left(\widehat{\mathbf{R}}_{H}(n), \mathbf{H}(n)\right)=-\log _{2}\left|\mathbf{I}+\frac{1}{\sigma_{w}^{2}} \mathbf{B B}^{H} \mathbf{H}^{H} \mathbf{H}\right|,
$$

where the transmission matrix $\mathbf{B} \in \mathbb{C}^{n_{T} \times n_{S}}$ is defined as

$$
\mathbf{B}\left(\widehat{\mathbf{R}}_{H}(n)\right)=\tilde{\mathbf{U}}(n) \mathbf{P}^{1 / 2}(n), \quad \mathbf{P}(n)=\operatorname{diag}\left(p_{1}, \ldots, p_{n_{S}}\right),
$$

and $\tilde{\mathbf{U}}(n)$ consists of $n_{S}$ columns that are the $n_{S}$ eigenvectors of $\widehat{\mathbf{R}}_{H}(n)$ associated to its $n_{S}$ maximum eigenvalues $\left\{\lambda_{i}\right\}_{i=1}^{n_{S}}$. The power $\mathbf{P}(n)$ is allocated according to the waterfilling solution $\left(p_{i}=\max \left\{0, \mu-1 / \lambda_{i}\right\}\right.$ where $\mu$ is a constant such that $\left.\sum_{i=1}^{n_{S}} p_{i}=P_{T}\right)$ [4].

The next sections are devoted to describe an algorithm for quantizing the actual correlation matrix $\mathbf{R}_{H}$ (instead of $\mathbf{H}$ ) from the receiver to the transmitter in a differential way. Since $\mathbf{R}_{H}$ belongs to the set of Hermitian positive definite matrices, ${ }^{2}$ exploiting its inherent geometry will improve the performance of the quantization.

\section{GEODESIC CURVES, DISTANCES AND SCALAR PRODUCTS}

As shown in [6] the set of Hermitian positive definite matrices $\mathcal{S}=$ $\left\{\mathbf{R} \in \mathbb{C}^{n_{T} \times n_{T}}: \mathbf{R}^{H}=\mathbf{R}, \mathbf{R} \succ \mathbf{0}\right\}$ is a convex cone ${ }^{3}$, i.e.,

\footnotetext{
${ }^{1}$ Note that the focus of this paper is not on the definition of the cost funcion, but on the minimization of any cost function that depends simultaneously on the estimated channel correlation matrix and the actual channel.

${ }^{2}$ In the following we will assume that the channel correlation matrix is strictly positive definite. If this cannot be guaranteed because, for example, $n_{R}<n_{T}$, it is possible to work with extended correlation matrices defined as $\widetilde{\mathbf{R}}_{H}=\mathbf{H}^{H} \mathbf{H}+\epsilon \mathbf{I}, \epsilon>0$, which are positive definite by construction.

${ }^{3}$ Actually, reference [6] is devoted to the case of real matrices, although the results and conclusions can be extended directly to the complex case.
}

$\forall \mathbf{R}_{1}, \mathbf{R}_{2} \in \mathcal{S}, \forall s \geq 0, \mathbf{R}_{1}+s \mathbf{R}_{2} \in \mathcal{S}$. The characterization of this set is described properly by means of differential geometry, which states a set of definitions for the distance, scalar products and routes within this set:

- Scalar product and norm: At any point in this set $\mathcal{S}$ given by $\mathbf{R}$ (also named as base point), the scalar product between two Hermitian matrices $\mathbf{A}$ and $\mathbf{B}$ is defined as $\langle\mathbf{A}, \mathbf{B}\rangle_{\mathbf{R}}=$ $\operatorname{Tr}\left(\mathbf{R}^{-1} \mathbf{A} \mathbf{R}^{-1} \mathbf{B}\right)$. This definition implies that the norm is defined as $\|\mathbf{A}\|_{\mathbf{R}}=\sqrt{\operatorname{Tr}\left(\mathbf{R}^{-1} \mathbf{A} \mathbf{R}^{-1} \mathbf{A}\right)}$.

- Geodesic curve: Let us take two points $\mathbf{R}_{1}$ and $\mathbf{R}_{2}$ in the set $\mathcal{S}$. Then, the geodesic curve $\Gamma(t)$, which is the curve connecting these points with minimum distance and with all its points belonging to $\mathcal{S}$, is given by

$$
\Gamma(t)=\mathbf{R}_{1}^{1 / 2} \exp (t \mathbf{C}) \mathbf{R}_{1}^{1 / 2},
$$

where $\mathbf{C}=\log \left(\mathbf{R}_{1}^{-1 / 2} \mathbf{R}_{2} \mathbf{R}_{1}^{-1 / 2}\right), \Gamma(0)=\mathbf{R}_{1}$, and $\Gamma(1)=\mathbf{R}_{2}$. The derivative of the geodesic curve at $t=0$, which is in fact the direction of such curve at $t=0$, is given by the Hermitian matrix $\Gamma^{\prime}(0)=\mathbf{R}_{1}^{1 / 2} \mathbf{C R}_{1}^{1 / 2}$.

- Geodesic distance: The geodesic distance between any two points in $\mathcal{S}$ is given by the length of the geodesic curve that connects them. According to the previous notation, it can be shown that this distance is given by

$$
\operatorname{dist}_{g}(\Gamma(0), \Gamma(t))=|t|\|\mathbf{C}\|_{F}, \Rightarrow \operatorname{dist}_{g}\left(\mathbf{R}_{1}, \mathbf{R}_{2}\right)=\|\mathbf{C}\|_{F} .
$$

or, using an equivalent expression,

$$
\operatorname{dist}_{g}\left(\mathbf{R}_{1}, \mathbf{R}_{2}\right)=\left(\sum_{i}\left|\log \lambda_{i}\right|^{2}\right)^{1 / 2}
$$

where $\left\{\lambda_{i}\right\}$ are the eigenvalues of matrix $\mathbf{R}_{1}^{-1 / 2} \mathbf{R}_{2} \mathbf{R}_{1}^{-1 / 2}$.

\section{ALGORITHM DESCRIPTION}

The fundamentals of the algorithm proposed in this paper are based on a differential quantization of the channel correlation matrix $\mathbf{R}_{H}(n)$. The objective is to minimize the cost function as presented in section 2, which can be related to the quality measure of the system. In this case the receiver has to know which is the kind of design that the transmitter will perform. If a more general setup is to be considered so that the feedback can be used for any transmitter design, a cost function that can be used is simply the geodesic distance between the actual channel correlation matrix and its fed back estimate, i.e., $d\left(\widehat{\mathbf{R}}_{H}(n), \mathbf{H}(n)\right)=\operatorname{dist}_{g}\left(\widehat{\mathbf{R}}_{H}(n), \mathbf{H}^{H}(n) \mathbf{H}(n)\right)$.

The differential quantization algorithm for the feedback of the channel correlation matrix is an iterative procedure, defined by the following steps corresponding to iteration $n$ :

- Initial situation: The receiver has a perfect knowledge of the current channel matrix $\mathbf{H}(n)$. Both the transmitter and the receiver know which is the last estimate of the channel correlation matrix sent through the feedback channel $\widehat{\mathbf{R}}_{H}(n-1)$. At the first iteration the algorithm starts from the cone vertex: $\widehat{\mathbf{R}}_{H}(0)=\mathbf{I}$.

- Step 1: Both the receiver and the transmitter generate a common set of $Q$ random Hermitian matrices using the same pseudo-random generator and the same seed. Then, these matrices are orthonormalized using the Gram-Schmidt procedure [7] according to the definition of scalar product presented in section 3 , producing the set $\left\{\mathbf{A}_{i}\right\}_{i=1}^{Q}$. The base 


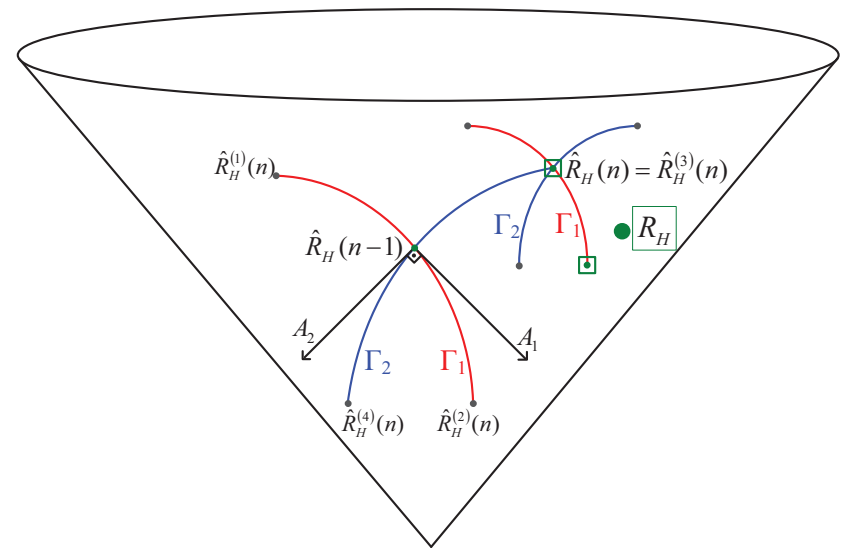

Fig. 1. 2-bit differential quantization in the space of channel correlation matrices.

point used in the scalar product is $\mathbf{R}=\widehat{\mathbf{R}}_{H}(n-1)$. Finally, each matrix $\mathbf{A}_{i}$ is re-scaled individually so that $\mathbf{C}_{i}=$ $\mathbf{R}^{-1 / 2} \mathbf{A}_{i} \mathbf{R}^{-1 / 2}$ has a norm equal to $\Delta\left(\left\|\mathbf{C}_{i}\right\|_{F}=\Delta\right)$. Note that this parameter $\Delta$ will be, in fact, the quantization step of the algorithm, and its impact on the algorithm performance will be studied in section 5 .

- Step 2: Both the receiver and the transmitter use the previous matrices to generate a set of $Q$ geodesic curves $\left\{\Gamma_{i}(t)\right\}_{i=1}^{Q}$ having all of them the same initial point $\mathbf{R}=\widehat{\mathbf{R}}_{H}(n-1)$ and with orthogonal directions:

$$
\Gamma_{i}(t)=\widehat{\mathbf{R}}_{H}^{1 / 2}(n-1) \exp \left(t \mathbf{C}_{i}\right) \widehat{\mathbf{R}}_{H}^{1 / 2}(n-1) .
$$

The maximum number of orthogonal routes is given by the dimension of the set of Hermitian matrices, i.e., $Q \leq n_{T}^{2}$.

- Step 3: Each of these geodesic curves is used to generate two candidates for the feedback in the next iteration $\widehat{\mathbf{R}}_{H}(n)$. Thus, the number of candidates will be $2 Q$ :

$$
\left\{\begin{aligned}
\widehat{\mathbf{R}}_{H}^{(2 i-1)}(n) & =\Gamma_{i}(-1), & & 1 \leq i \leq Q, \\
\widehat{\mathbf{R}}_{H}^{(2 i)}(n) & =\Gamma_{i}(1), & & 1 \leq i \leq Q .
\end{aligned}\right.
$$

- Step 4: The receiver evaluates the cost function for each of the candidates, and sends the corresponding index $i_{F B}$ through the feedback channel to the transmitter (therefore, the number of feedback bits has to be higher than or equal to $\left.\log _{2}(2 Q)\right)$. The selected matrix will be used for the transmitter design and as the starting point in the next iteration:

$$
\begin{aligned}
i_{F B} & =\arg \min _{i} d\left(\widehat{\mathbf{R}}_{H}^{(i)}(n), \mathbf{H}(n)\right), \quad 1 \leq i \leq 2 Q, \\
\widehat{\mathbf{R}}_{H}(n) & =\widehat{\mathbf{R}}_{H}^{\left(i_{F B}\right)}(n)
\end{aligned}
$$

Figure 1 shows the differential quantization process using 2 bits. Starting from $\widehat{\mathbf{R}}_{H}(n-1)$, the algorithm generates 2 geodesic routes $\Gamma_{1}(t)$ and $\Gamma_{2}(t)$ with orthogonal velocity matrices $\mathbf{A}_{1}$ and $\mathbf{A}_{2}$, respectively. The four quantization candidates are: $\widehat{\mathbf{R}}_{H}^{(1)}(n)=\Gamma_{1}(-1), \widehat{\mathbf{R}}_{H}^{(2)}(n)=\Gamma_{1}(1), \widehat{\mathbf{R}}_{H}^{(3)}(n)=\Gamma_{2}(-1)$, and $\widehat{\mathbf{R}}_{H}^{(4)}(n)=\Gamma_{2}(1)$. At the receiver, each candidate is compared to the actual $\mathbf{R}_{H}$ and the one with smallest cost function (in this example candidate 3 ) is chosen. That is, its index $i_{F B}=3$ is sent to the transmitter through the feedback channel and $\widehat{\mathbf{R}}_{H}(n)=\widehat{\mathbf{R}}_{H}^{(3)}(n)$.

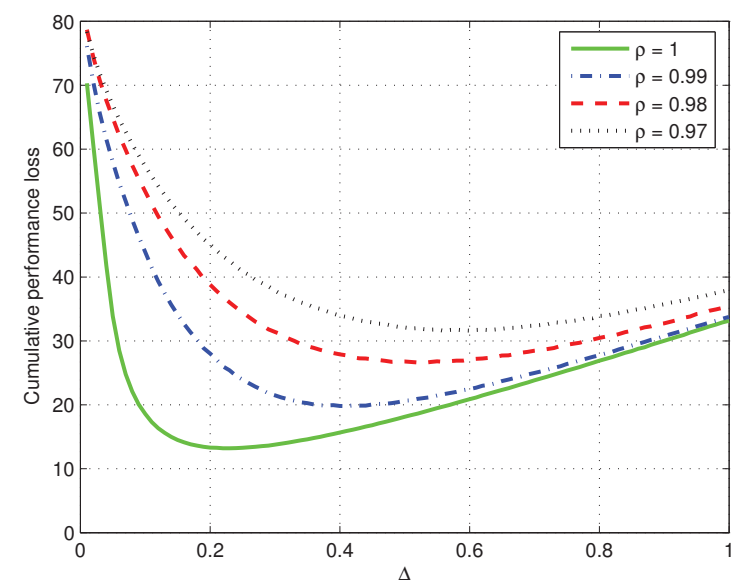

Fig. 2. Cumulative performance loss versus quantization step $\Delta$.

The next iteration starts from this point, generates 2 orthogonal routes and 4 quantization candidates, selects the closest candidate to $\mathbf{R}_{H}$ (according to the cost function), and so on.

\section{SIMULATION RESULTS}

In the simulations, we consider a random MIMO channel following a first-order time-variation model according to the expression:

$$
\mathbf{H}(n)=\rho \mathbf{H}(n-1)+\sqrt{1-\rho^{2}} \mathbf{N}(n),
$$

where matrices $\mathbf{H}(n-1)$ and $\mathbf{N}(n)$ are assumed to be independent and composed of i.i.d. zero-mean complex Gaussian entries with unit variance. The time correlation factor $\rho$ models the variability of the channel and depends on the Doppler frequency of the terminal $f_{D}$ through the expression $\rho=J_{0}\left(2 \pi f_{D} t_{d e l}\right)$ [8], where $J_{0}$ is the zeroth-order Bessel function of the first kind and $t_{d e l}$ corresponds the time difference between consecutive feedback instants. The case of an invariant channel corresponds to $\rho=1$.

The quantization step $\Delta$, presented in section 4 , is the geodesic distance between the last value of the estimated correlation matrix $\widehat{\mathbf{R}}_{H}(n-1)$ and the quantization candidates. The choice of this parameter has a direct effect on the behavior of the algorithm: larger values of $\Delta$ result in faster convergence but larger stable-state error, and smaller values of $\Delta$ result in slower convergence but also smaller stable-state error. In order to achieve a compromise between convergence rate and stable-state error, the optimization of $\Delta$ is made for minimum cumulative performance loss in a window of 50 iterations. That is, for each of the 50 first iterations the difference between the performance with perfect CSI and the performance using our limited feedback algorithm is added, and the result is optimized by the parameter $\Delta$. Figure 2 shows the cumulative performance loss versus $\Delta$ for different values of the correlation factor $\rho$. The results correspond to a setting of $n_{T}=2, n_{R}=2$ antennas, 3 feedback bits, a window of 50 iterations and an average over 200 channel realizations. As cost function we used the geodesic distance to the correlation matrix of the exact channel realization. The curves show how the optimum $\Delta$ increases as the MIMO channel is more timevariant (smaller $\rho$ ). For the rest of the simulations in this section the optimum $\Delta$ was taken for each case following this scheme.

Figure 3 shows the result of a simulation where the channel is constant and the performance is measured using the SNR criterion. The parameters taken in the simulation are $P_{T}=0.3, n_{T}=2$, $n_{R}=8$ antennas, 3 feedback bits and an average over 200 channel 


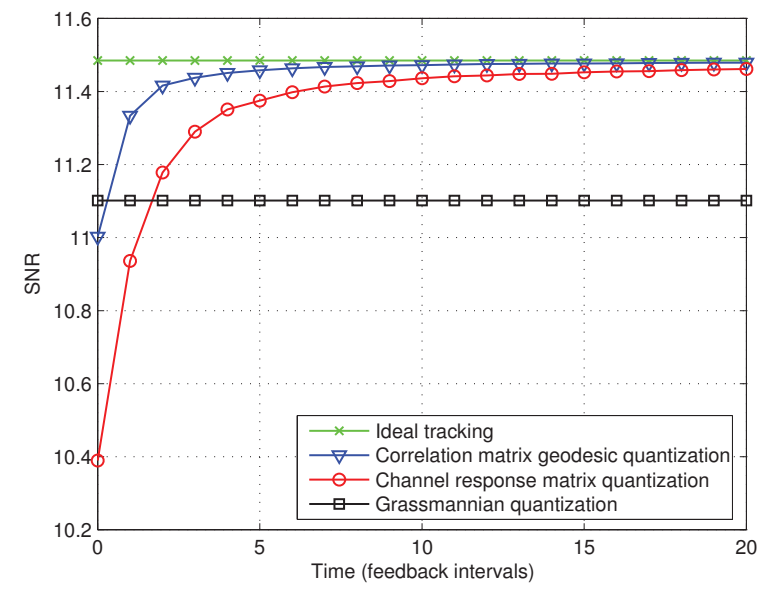

Fig. 3. SNR achieved using different feedback techniques in a constant channel.

realizations. Here the comparison is between the proposed tracking algorithm applied to the channel correlation matrices, a tracking algorithm of channel response matrices ${ }^{4}$, a non-differential quantization using Grassmannian packages [1] and ideal tracking using perfect CSI at the transmitter.

Our algorithm showed faster convergence and smaller stablestate error than the channel response matrix tracking. The gain is due to the fact that we use geodesic routes on the space of correlation matrices and that in this case a quantization on $\mathbf{R}_{H}$ has less dimensions than a quantization on $\mathbf{H}$ ( $\mathbf{R}_{H}$ is Hermitian, positive definite and $2 \times 2$, while $\mathbf{H}$ is random Gaussian $8 \times 2$ ). The non-differential Grassmannian technique presents a floor in the performance since it does not exploit the time-invariance of the channel.

The behavior in a time variant channel of the algorithm proposed is analyzed and compared to the algorithm from [3]. Figure 4 shows the performance of the two algorithms compared to that of an ideal tracking (perfect CSI) according to a mutual information criterion. The simulation considers a random realization of a channel with $\rho=0.97$ and $n_{T}=3, n_{R}=3$ antennas. 500 realizations of the algorithms over that channel realization were performed using the optimum quantization steps for each algorithm, $P_{T}=0.3$, $n_{S}=2$ streams and 1 feedback bit. Our algorithm offers a better performance than the algorithm in [3] because it tracks the eigenvectors and the eigenvalues of $\mathbf{R}_{H}$, allowing a waterfilling-like power allocation instead of the uniform power allocation used in [3], where the eigenvalues are unknown.

\section{CONCLUSIONS}

The feedback strategy proposed for MIMO communications, which is based on a differential quantization of the channel correlation matrix using geodesic routes, has several advantages over other existing feedback strategies. This technique exploits the intrinsic geometry of correlation matrices (positive definite Hermitian) versus channel response matrices in order to improve quantization performance. Furthermore, the use of orthogonal geodesic routes generates quantization candidates at each iteration that are better distributed than in the

\footnotetext{
${ }^{4}$ The tracking algorithm of the channel response matrices is also a differential quantization strategy applied to $\mathbf{H}(n)$ instead of $\mathbf{R}_{H}(n)$, where the quantization candidates are also obtained through orthogonal routes. Note that in this case, the orthogonal routes are simple straight lines and that the quantization step has also been optimized in order to maximize the performance and for a fair comparison with our proposal.
}

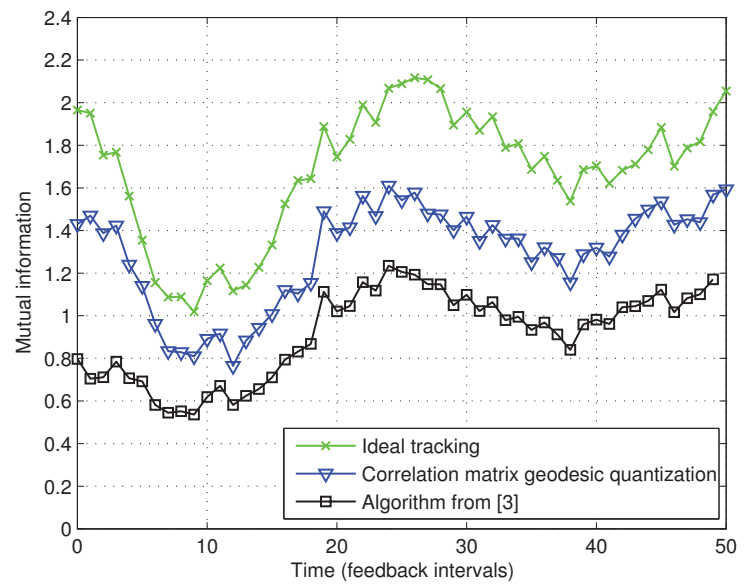

Fig. 4. Correlation matrix geodesic quantization and algorithm proposed in [3] compared to ideal tracking in a time variant channel.

case of channel response matrix quantization. This also leads to an improvement in the quantization performance. Another fundamental advantage lies on the fact that the transmitter is not forced to apply uniform power allocation among the eigenmodes, which translates into a design gain.

Simulations show that this algorithm achieves better performance than other techniques based on the direct quantization of the channel response matrix or the quantization of the subspace spanned by the strongest eigenmodes of the MIMO channel, as well as non-differential strategies like Grassmannian packaging.

\section{REFERENCES}

[1] D.J. Love, R.W. Heath, and T. Strohmer, "Grassmannian Beamforming for Multiple-Input Multiple-Output Wireless Systems," IEEE Trans. on Information Theory, vol. 49, no. 10, pp. 27352747, October 2003.

[2] A. Edelman, T.A. Arias, and S.T. Smith, "The Geometry of Algorithms with Orthogonality Constraints," SIAM J. Matrix Anal. Appl., vol. 20, no. 2, pp. 303-353, October 1998.

[3] J. Yang and D.B. Williams, "Transmission Subspace Tracking for MIMO Systems With Low-Rate Feedback," IEEE Trans. on Communications, vol. 55, no. 8, pp. 1629-1639, August 2007.

[4] D.P. Palomar, J.M. Cioffi, and M.A. Lagunas Hernández, "Joint Tx-Rx Beamforming Design for Multicarrier MIMO Channels: a Unified Framework for Convex Optimization," IEEE Trans. on Signal Processing, vol. 51, no. 9, pp. 2381-2401, September 2003.

[5] J.J. Boutros, F. Kharrat-Kammoun, and H. Randriambololona, "A Classification of Multiple Antenna Channels," in Proc. IEEE International Zurich Seminar on Communications (IZS'06), February 2006, pp. 14-17.

[6] M. Talih, "Geodesic Markov Chains on Covariance Matrices," Tech. Rep., Statistical and Applied Mathematical Sciences Institute, March 2007.

[7] G.H Golub and C.F. Van Loan, Matrix Computations, Third Edition, The Johns Hopkins University Press, 1996.

[8] R. Steele and L. Hanzo, Eds., Mobile Radio Communications, John Wiley \& Sons, second edition, 1999. 